\title{
UMA PROPOSTA DE ATIVIDADE MATEMÁTICA SOBRE O TEMPO DE EXPOSIÇÃO AO SOL
}

\section{AN EMPLOYEMNT OF MATHEMATICAL MODELING ON THE TIME OF EXPOSURE TO THE SUN}

\author{
Kattia Ferreira da Silva ${ }^{1}$ \\ ORCID iD: 0000-0002-4154-179X
}

Raylson dos Santos Carneiro ${ }^{2}$

ORCID iD: 0000-0002-4571-5822

Rogerio dos Santos Carneiro ${ }^{3}$

ORCID iD: 0000-0002-5387-0435

\section{RESUMO}

A necessidade de pensar novas metodologias para o ensino da matemática, especialmente a partir de temas relacionados ao cotidiano dos alunos, motivou a realização desta pesquisa, que tem como objetivo aliar teoria e prática no contexto da educação básica, para verificar o tempo que uma pessoa, fazendo uso adequado do fator de proteção solar (FPS) 30, pode ficar exposta à radiação ultravioleta (UV) do sol, sem sofrer queimaduras. Por meio da modelagem matemática, apresenta-se uma proposta para o ensino de matemática, que tende a permitir ao professor do ensino médio tratar conceitos de função exponencial de maneira crítica e reflexiva, para a construção de um conhecimento mais significativo e também fornecer aos alunos condições de perceber a integração da matemática com outras áreas. Ao considerar os níveis de radiação, o FPS 30 em relação ao tipo de pele, os dados remetem a uma função exponencial, que, ao se aplicar a metodologia do método dos mínimos quadrados, viabilizou elaborar um modelo matemático para cada um dos quatro tipos de pele. Os resultados mostram que, se as pessoas fizerem o uso correto do FPS 30, a depender do tipo de pele, é possível permanecer em exposição ao sol por um tempo mínimo de $1 \mathrm{~h}$ e 45 minutos, sem sofrer queimaduras.

Palavras-chave: Modelagem Matemática. Fator de Proteção Solar. Ensino de Matemática.

\begin{abstract}
The need to think about new methodologies for teaching mathematics, especially from themes related to students' daily lives, motivated this research, which aims to combine theory and practice in the context of basic education, to verify the time that a person, making appropriate use of the sun protection factor

\footnotetext{
${ }^{1}$ Mestre em Matemática, Mestrado Profissional em Matemática em Rede Nacional (PROFMAT), Universidade Federal do Tocantins (UFT). Professora efetiva da Universidade de Gurupi - UNIRG, campus de Gurupi. E-mail: kattia-silva@hotmail.com.

${ }^{2}$ Mestre em Matemática, Mestrado Profissional em Matemática em Rede Nacional (PROFMAT), Universidade Federal do Tocantins (UFT). Professor da Engenharia Florestal, Universidade Federal do Tocantins (UFT), campus de Gurupi. E-mail: raylsonscarneiro@hotmail.com.

${ }^{3}$ Doutorando em Educação em Ciências e Matemática, Programa de Doutorado em Educação em Ciências e Matemática (PPGECEM), Universidade Federal do Mato Grosso (UFMT). Professor da Licenciatura em Matemática, Universidade Federal do Tocantins (UFT), campus de Araguaína. E-mail: rogerioscarneiro@gmail.com.
} 
(SPF) 30, can be exposed to ultraviolet (UV) radiation from the sun, without suffering burns. Through mathematical modeling, a proposal for the teaching of mathematics is presented, which tends to allow high school teachers to treat exponential function concepts in a critical and reflective way, to build more meaningful knowledge and also provide students with able to perceive the integration of mathematics with other areas. When considering the radiation levels, the SPF 30 in relation to the skin type, the data refer to an exponential function, which, by applying the least squares method methodology, made it possible to develop a mathematical model for each of the four types of skin. The results show that, if people make the correct use of SPF 30, depending on the type of skin, it is possible to remain in the sun for a minimum of 1 hour and 45 minutes, without suffering burns.

Keywords: Mathematical Modelling. Sun Protection Factor. Mathematics Teachingord.

\section{INTRODUÇÃO}

Uma vez que, atualmente, há uma tendência a inovações curriculares ou inovações nas práticas voltadas ao ensino da matemática na educação básica, muitos professores têm ido em busca de alternativas para tornar esse ensino atrativo. De acordo com Bassanezi (2011), a Modelagem Matemática é entendida como uma metodologia de ensino, que tende a transformar problemas do cotidiano dos alunos em problemas matemáticos e resolvê-los, interpretando suas soluções na linguagem do mundo real. Isso permite, de certo modo, compreender a importância da matemática e sua aplicabilidade no dia a dia.

Assim, a modelagem matemática se constitui numa ferramenta capaz de propiciar uma aprendizagem mais significativa, já que essa metodologia de ensino torna possível o desenvolvimento de habilidades matemáticas, ao conectar a realidade do educando ao estudado em sala de aula. Ela possibilita fazer com que a aprendizagem dos conceitos e/ou conteúdos possa acontecer de forma consistente, pois oportuniza ao discente reconhecer que pode usar a matemática no meio em que vive, na sua realidade local.

O problema da pesquisa que orienta este estudo focaliza um tema, cuja compreensão envolve diferentes áreas de conhecimento, permitindo, assim, um trabalho transdisciplinar e colaborativo entre os professores. Como o Brasil é um país com bastante incidência de luz solar, esse fato instigou o presente estudo acerca dos efeitos da radiação solar sobre a pele humana.

Este artigo é oriundo de um trabalho, embasado numa investigação documental de cunho bibliográfico, pesquisa bibliográfica, realizada por meio de livros, revistas especializadas, artigos ou sites especializados, com o intuito de obter respaldo teórico para fundamentar a pesquisa sobre o tempo de exposição ao sol, o uso do FPS 30 e a representação 
das variáveis em estudo a partir dos conceitos da modelagem matemática em consonância com o uso do método dos mínimos quadrados.

A princípio não se tinha noção de que as variáveis em estudo iriam resultar no estudo de funções exponenciais. Contudo, somente após a disposição dos dados no plano cartesiano, foi visualizado que os dados em estudo resultariam nesse tipo de função. Gomes (2007, p. 91) anuncia que "chegamos a uma interpretação quando conseguimos realizar uma síntese entre: as questões da pesquisa, os resultados obtidos a partir da análise do material coletado, as inferências realizadas e a perspectiva teórica adotada".

Isso posto, esta pesquisa, objetiva, de modo geral, discutir e apresentar, por meio da elaboração de modelos matemáticos - metodologia de ensino da modelagem matemática -, uma proposta que alia a teoria e a prática no contexto da educação básica, para verificar o tempo que uma pessoa, fazendo uso adequado do fator de proteção solar (FPS) 30, pode ficar exposta à radiação ultravioleta (UV) do sol, sem sofrer queimaduras.

\section{MODELAGEM MATEMÁTICA SOBRE EXPOSIÇÃO AO SOL COM APLICAÇÃO DO MÉTODO DOS MÍNIMOS QUADRADOS}

O Brasil é um país que está localizado em uma região que recebe grande intensidade de radiação solar. No entanto, o hábito de se proteger dos efeitos dos raios ultravioletas com a utilização de filtro solar ou outras formas de proteção ainda é baixo.

Segundo Schalka (2009, p. 10), “durante o segundo Congresso internacional e Luz, em Copenhagen, Dinamarca, em 1932, Willian Coblents propôs a divisão do espectro ultravioleta do sol em três regiões espectrais: UVA (315 - $400 \mathrm{~nm})$, UVB (280 - $315 \mathrm{~nm})$ e UVC ( < 280 nm)”, ou seja, a radiação ultravioleta (UV) é categorizada em UVA, UVB e UVC. A diferença entre elas se deve, principalmente, ao histórico de exposição da pessoa e a diferentes comprimentos de onda da radiação UV, uma vez que a radiação UVB é cerca de 1.000 vezes mais "agressiva" do que a radiação UVA. Essa diferença faz com que a radiação UVA tenha uma contribuição de somente $15 \%$ a $20 \%$ na quantidade de energia responsável pela queimadura/eritema ${ }^{4}$. Uma vez filtrada pela atmosfera, a radiação UVC não atinge a superfície da Terra.

\footnotetext{
${ }^{4}$ Eritema, sinal clínico presente em várias patologias, é caracterizado pela presença de lesões avermelhadas na pele. Ele pode ter caráter transitório ou permanente e ser causado por uma série de fatores externos (calor, fricção, luz solar, irritações químicas ou por picada de insetos, etc.) ou internos (reflexos vasomotores de origem digestiva, medicamentosa, nervosa, psíquica, vascular, etc.). 
O índice ultravioleta (IUV) é um parâmetro criado para definir a intensidade da radiação ${ }^{5}$ a que uma pessoa está exposta, no caso a radiação do sol na faixa UVB, e foram definidos 15 degraus de intensidade: 0 a 2 - baixo nível de radiação; 3 a 5 - nível moderado de radiação; 6 a 7 - alto nível de radiação; 8 a 10 - nível muito alto de radiação; e 11 a 15 - nível extremo de radiação, de acordo com Kirchhoff (1995). O IUV atinge nível pleno das 10h às 16h, no momento em que as temperaturas alcançam o seu índice mais alto. Historicamente o pico do IUV é registrado ao meio-dia. Sendo que o índice 15 equivale ao mais intenso, correspondente ao pico de verão.

A indústria farmacêutica tem produzido uma série de produtos para proteger a pele dos efeitos da radiação solar. Trata-se de substâncias que, aplicadas sobre a pele, funcionam como barreira contra a ação dos raios ultravioleta. A eficácia desses protetores solares é definida pela sua capacidade de proteger a pele contra a queimadura solar e reduzir o acúmulo de todos os danos provocados pela radiação ultravioleta, conforme Milese e Guterres (2002).

De acordo com Matos (2014), para que o usuário do protetor solar tenha conhecimento da capacidade de proteção do cosmético, é necessário estar atento ao fator de proteção solar (FPS) que ele diz oferecer. $\mathrm{O}$ valor numérico que acompanha essa sigla indica quantas vezes mais, em relação ao tempo, o usuário com esse cosmético sobre a pele está protegido, ou seja, um protetor com FPS 10 indica que seu usuário estará 10 vezes mais protegido, em relação ao tempo, do que se estivesse sem protetor. Tem-se aqui, então, o modelo matemático de uma função linear $f(x)=F P S . x$, sendo que FPS é o fator de proteção solar, e a variável $x$ que representa o tempo de exposição sem nenhuma proteção, característico de cada tipo de pele.

Ainda segundo Matos (2014), esse tempo existe porque cada pessoa tem um tempo de proteção natural, resultante da quantidade de melanina. Apenas depois desse tempo é que começamos a sofrer os danos da exposição ao sol. Então, considerando que uma pessoa, cujo tempo de proteção natural é de 12 minutos, caso ela utilize um protetor FPS 10, ela ficará protegida durante 120 minutos. Teoricamente, somente depois desse tempo seria necessária a reaplicação do produto.

Uma vez que os valores de FPS estão relacionados ao tempo de permanência sob o sol, entende-se que quanto maior o FPS, maior será o tempo de proteção. Ocorre que o aumento no percentual de proteção não é proporcional ao número do FPS. Para facilitar o entendimento, observe os dados contidos na Tabela 1.

${ }^{5}$ Mais informacões sobre os níveis de radiação do sol. consultar Rouessac e Rouessac (2000). 
Tabela 1 - Capacidade de proteção de acordo com o FPS

\begin{tabular}{c|c|c|c|c|c|c|c|c|c}
\hline FPS & 2 & 4 & 8 & 15 & 20 & 25 & 30 & 40 & 50 \\
\hline Proteção (\%) & 50 & 75 & 87,5 & 93,3 & 95 & 96 & 96,6 & 97,5 & 98 \\
\hline
\end{tabular}

Ao observarmos os dados da Tabela 1, mesmo duplicando o valor do FPS (15, para 30, por exemplo), a capacidade de proteção não aumenta na mesma proporção (FPS 15 = 93,3 e FPS 30 =96,6). De outro modo, ao utilizar um cosmético com FPS 15, teremos cerca de 6,7\% de radiação UVB entrando na nossa epiderme, ao passo que, ao utilizar FPS 30, será cerca de $3,4 \%$. O tempo de exposição permissível ao sol, sem queimar, foi obtido a partir de experimentos. Já que o índice UVB é o que requer mais atenção em relação aos três tipos de radiação solar, é dele que trataremos a seguir.

Caracterizar um determinado tipo de pele, em tese, é um processo subjetivo e muito difícil devido à miscigenação e às diferentes características entre os seres humanos. Cada pessoa possui um biótipo próprio, inclusive por conta da quantidade de melanina, e cada um responde de maneira específica a um dado nível de exposição ao sol. Para este estudo, utilizamos a classificação do tipo de pele proposta por Fitzpatrick e Mosher (1983), que apresentam a pele humana em quatro categorias básicas, em relação à cor e ao efeito de bronzeamento e/ou queimadura produzido pela radiação solar UVB.

- Tipo A: Pele muito branca, a mais sensível.

- Tipo B: Pele moreno clara.

- Tipo C: Pele moreno escura.

- Tipo D: Pele negra, a menos sensível.

Levando em conta o tipo de pele (A, B, C e D), tem-se, na Tabela 2, a indicação do tempo (em minutos) que uma pessoa, exposta ao sol com a pele desprotegida, pode suportar sem que haja danos de queimadura (eritema), provocados pela radiação solar.

Tabela 2: Tempo médio (em minutos) de exposição segura ao sol (UVB)

\begin{tabular}{c|c|c|c|c|c|c|c|c|c|c|c|c|c|c}
\hline $\begin{array}{l}\text { Valor do } \\
\text { Indice UVB }\end{array}$ & $\mathbf{0 - 2}$ & $\mathbf{3}$ & $\mathbf{4}$ & $\mathbf{5}$ & $\mathbf{6}$ & $\mathbf{7}$ & $\mathbf{8}$ & $\mathbf{9}$ & $\mathbf{1 0}$ & $\mathbf{1 1}$ & $\mathbf{1 2}$ & $\mathbf{1 3}$ & $\mathbf{1 4}$ & $\mathbf{1 5}$ \\
\hline Pele Tipo A & 30 & 20 & 15 & 12 & 10 & 8,5 & 7,5 & 7 & 6 & 5,5 & 5 & 4,5 & 4 & 3,5 \\
\hline Pele Tipo B & 60 & 47 & 32 & 25 & 22 & 19 & 17 & 15 & 14 & 13 & 12 & 11 & 10 & 9 \\
\hline Pele Tipo C & 90 & 70 & 50 & 40 & 35 & 30 & 26 & 24 & 22 & 20 & 19 & 18 & 17 & 15 \\
\hline Pele Tipo D & 120 & 90 & 75 & 60 & 50 & 40 & 35 & 33 & 30 & 27 & 25 & 23 & 21 & 20 \\
\hline \multicolumn{10}{c}{ Fonte: Laboratório de Ozônio do INPE (2012). }
\end{tabular}

Fonte: Laboratório de Ozônio do INPE (2012). 
Embora haja diversos valores numéricos de FPS, neste estudo tomamos como referência o FPS 30. Tal opção se deu em função de que, segundo o Food and Drug Administration (FDA), (apud SCHALKA, 2009), órgão americano que fiscaliza alimentos e medicamentos, quando aplicado corretamente o protetor oferece quase $97 \%$ de proteção. O tempo de bloqueio ao sol, oferecido por esse produto, já seria mais do que suficiente, mesmo porque nos FPS acima de 30, a proteção seria irrisória sem mencionar que nunca alcançaria 100\%.

\section{1 Emprego da modelagem matemática na educação básica}

A Lei Federal 9394/96, ao fixar as Diretrizes e Bases da Educação Nacional (LDBEN), explicita que a educação escolar tem por finalidade o pleno desenvolvimento do educando, seu preparo para o exercício da cidadania e sua qualificação para o trabalho (BRASIL, 1996), sendo assim deverá vincular-se ao mundo do trabalho e à prática social. Um ambiente escolar que atenda à função social busca um ensino da matemática que assegure a participação dos educandos como os principais partícipes do processo, que transforme a aprendizagem mecânica numa aprendizagem significativa, seguindo o pressuposto legal.

A modelagem matemática é uma alternativa pedagógica que, por meio da problematização, instiga os alunos e possibilita discutir questões do dia a dia, com o auxílio também da matemática. Bassanezi (2011) conta que a modelagem matemática, como metodologia de ensino, surgiu na sala de aula mais por necessidade do que por acaso, pois a elaboração de modelos, por muitas vezes, se torna mais atrativa do que a sua própria resolução. Isso porque, "a criação de modelos matemáticos vem ao encontro da necessidade de que se desenvolva uma técnica de acesso ao conhecimento mais dinâmica, mais realista e menos formal, mesmo no ensino tradicional, permitindo atingir objetivos mais adequados a nossa realidade" (D'AMBROSIO, 1986, p. 25).

Na educação básica, a modelagem matemática, quando desenvolvida, pode favorecer a construção do conhecimento matemático, posto que potencializa uma aprendizagem significativa, uma vez que, a partir de um fato real, se pode criar, por meio da coleta, da organização dos dados e da análise, uma expressão em linguagem matemática capaz de servir de parâmetro para descrição e compreensão da realidade pelo modelo criado.

No tocante ao ensino da matemática, a modelagem se configura como um processo significante, pois "[...] é uma perspectiva de educar matematicamente, que vai problematizar também o currículo e usar as ferramentas matemáticas para aquele tipo de problema específico, 
que está sendo investigado naquele momento" (MEYER; CALDEIRA; MALHEIROS, 2013, p. 40-41).

Para Biembengut e Hein (2009), a importância da adoção deste método de ensino reside tanto na possibilidade de despertar no aluno o interesse por tópicos matemáticos que ele ainda desconhece, quanto na apresentação de conhecimentos necessários para que o aluno aprenda a arte de modelar matematicamente.

O processo envolvido na modelagem matemática acontece por etapas, trata-se de uma espécie de sistematização que caracteriza a ação como modelagem. Assim sendo, "o primeiro passo a ser dado para se trabalhar com modelagem é reconhecer a existência de um problema real, no sentido de ser significativo para os alunos e suas comunidades" (MEYER; CALDEIRA; MALHEIROS, 2013, p. 27). Ainda segundo os mesmos autores, podemos simplificar o processo de modelagem em educação matemática em cinco momentos, detalhados a seguir:

1. Determinar a situação: momento de analisar quais situações-problema existentes no mundo real possuem maior significação para quem pretende se utilizar da modelagem. Assim elas são avaliadas e olhadas em uma perspectiva crítica e, a partir daí, é escolhida qual delas será trabalhada.

2. Simplificar as hipóteses dessa situação: momento de conhecer o problema a fundo, com o intuito de definir estratégias de simplificação. Isso é feito, muitas vezes, a fim de facilitar a resolução matemática ou, até mesmo, de colocar o problema no nível dos alunos. Não se trata de simplificar o problema real, mas sim, de introduzir hipóteses que simplifiquem sua abordagem, procurando, dessa maneira, traduzir o problema para uma linguagem do universo matemático.

3. Resolver o problema matemático decorrente: momento de considerar que, por se tratar de uma situação real, os dados obtidos exigem aproximações, algoritmos e avaliação das respostas matemáticas. Esta situação será analisada com base nas ferramentas matemáticas de que se dispõe.

4. Validar as soluções matemáticas de acordo com a questão real: momento de legitimar a solução obtida em termos do problema que gerou a questão anterior, ou seja, a solução deve atender às exigências da situação.

5. Definir a tomada de decisão com base nos resultados: momento de conhecer as dificuldades de agir em sociedade e a necessidade de fazê-lo, já que se trata de modelagem voltada para a educação. 
Se o modelo não atende ao problema estudado, será necessário modificá-lo, voltando às etapas anteriores para encontrar as inconsistências que levaram aos erros identificados nas respostas desse modelo, caracterizando assim a etapa de Modificação (MENEZES; BRAGA; SANTO, 2019). Ao utilizar a metodologia de ensino modelagem matemática, faz-se importante estar atento aos cinco momentos, apresentados anteriormente, para o desenvolvimento de uma atividade, pois, além do aprendizado de conteúdos matemáticos, ela propicia colaborar com a formação de pessoas capazes de evidenciar o papel da matemática e intervir na sociedade em que vivem. Isso porque, a

\footnotetext{
Modelagem Matemática é um processo dinâmico utilizado para a obtenção e validação de modelos matemáticos. É uma forma de abstração e generalização com a finalidade de previsão de tendências. A modelagem consiste, essencialmente, na arte de transformar situações da realidade em problemas matemáticos cujas soluções devem ser interpretadas na linguagem usual. (BASSANEZI, 2004, p. 24)
}

Ao sugerir uma proposta de modelagem matemática neste trabalho, não temos a intenção de prescrever uma receita, ou de apresentar a modelagem como uma única direção a ser seguida, mas sim, se valer dessa metodologia para, baseando-se numa situação real - tempo de exposição ao sol e o uso do protetor solar - relacionar o aprendizado da matemática com situações do cotidiano do aluno, tendo sua participação ativa e direta, e enfatizar, assim, a relevância do conhecimento matemático para a vida e para sua contribuição na construção da sociedade.

Neste estudo, será utilizado o método dos mínimos quadrados para se chegar ao modelo pretendido, mas cabe ao professor decidir qual o melhor método a ser adotado para que os alunos possam confeccionar o modelo. Atualmente, a maioria das calculadoras científicas já tem o programa de ajustes de curvas. O software Excel, um dos aplicativos do pacote Microsoft Office, pode ser um excelente programa a ser utilizado nas aulas, pois é encontrado em grande parte dos computadores utilizados no Brasil e pertence ao leque de tecnologias que contribuem, em vários aspectos, para o trabalho com a modelagem, porque facilita desde a coleta de dados, a organização e as tabulação desses dados, além de fazer simulações, traçar gráficos e prever tendências. Dessa forma, diante de todo o avanço tecnológico, é primordial repensar qual o objetivo da criação dos modelos, pois a matemática não mais deve ser estudada de maneira mecânica, já que o foco está na análise dos resultados e não na aplicação de procedimentos padrões. 


\subsection{Método dos mínimos quadrados}

O método dos mínimos quadrados não é, em geral, apresentado no ensino médio, pois demandaria o uso de derivada parcial, um assunto normalmente trabalhado no curso de nível superior. Barbosa e Breitschaft (2006) sugerem que, para o ensino médio, o método de mínimos quadrados seja substituído pelo ajuste visual de uma reta aos pontos experimentais, o que possibilita a determinação dos parâmetros desta reta. Tal modelo permitiria apresentar o método de mínimos quadrados no ensino médio.

Em diversos ramos da ciência, é comum tentar descobrir se existe uma relação matemática entre duas ou mais grandezas. O método dos mínimos quadrados é um processo que procura determinar a equação matemática mais adequada para definir a relação entre duas variáveis. Para estabelecer uma equação que represente um fenômeno em estudo, pode-se fazer um gráfico (diagrama de dispersão), para verificar como se comportam os valores da variável dependente $(y)$ em função da variação da variável independente $(x)$.

Entretanto, os dados em estudo dificilmente atendem a uma única função. $O$ comportamento da variável dependente $(y)$ em relação a variável independente $(x)$ pode ser apresentado de diversas maneiras: linear, quadrático, cúbico, exponencial, logarítmico, hiperbólico, geométrico, entre outros. Para se estabelecer o modelo para explicar o fenômeno em estudo, devemos verificar a qual tipo de curva os dados dispostos num gráfico (diagrama de dispersão) mais se aproximam.

\footnotetext{
Em termos de modelagem matemática de fenômenos caracterizados por um processo dinâmico, a formulação do modelo pode muitas vezes preceder à análise dos dados experimentais. Nestes casos, o método de ajuste de curvas é fundamental para a validação dos modelos estabelecidos a priori. [...] Em geral, o modelo depende de parâmetros e sua validação exige a estimação destes parâmetros, de modo que a curva (solução do modelo) ajustada represente, o mais próximo possível, o fenômeno estudado. (BASSANEZI, 2011, p. 56)
}

De acordo com Bassanezi, o método utilizado neste estudo para o desenvolvimento do modelo matemático da atividade proposta para o ensino médio é um dos métodos mais usados na estimação de parâmetros ou ajuste curvas. Os passos apresentados a seguir da descrição do método dos mínimos quadrados são adaptados de Bassanezi (2011, p. 57-63).

Considere um conjunto de $n$ dados observados $\left\{\bar{x}_{i}, \bar{y}_{i}\right\}$, onde $i=1,2,3, \ldots, n$ e uma função $y(x)=f\left(x ; a_{1}, a_{2}, \ldots, a_{k}\right)$, onde $a_{j}$, com $j=1, \ldots, k$ são os parâmetros, o Método dos 
Mínimos Quadrados consiste em determinar estes parâmetros de modo que "minimize" o valor de:

$$
S=\sum_{i=1}^{n}\left(y_{i}-\bar{y}_{i}\right)^{2}=\sum_{i=1}^{n}\left[f\left(\bar{x}_{i} ; a_{1}, \ldots, a_{k}\right)-\bar{y}_{i}\right]^{2},
$$

Isto é, devemos minimizar a soma dos quadrados dos desvios entre os valores $\bar{y}_{i}$ observados e os valores $y_{i}=f\left(\bar{x}_{i} ; a_{1}, \ldots, a_{k}\right)$ ajustados.

Começaremos pela fundamentação teórica de um ajuste linear; em seguida trataremos do ajuste exponencial, que é objeto desse estudo.

Um ajuste é linear se for da forma:

$$
y(x)=f(x ; a, b)=a x+b \quad(\text { equação de uma reta })
$$

Neste caso, devemos encontrar os valores dos parâmetros $a$ e $b$ que tornam mínimo o valor da soma dos quadrados dos desvios:

$$
S=S(b, a)=\sum_{i=1}^{n}\left(b+a \bar{x}_{i}-\bar{y}_{i}\right)^{2}
$$

Tais valores devem satisfazer, necessariamente, as condições:

$$
\left\{\begin{array}{l}
a=\frac{n \sum \bar{x}_{i} \bar{y}_{i}-\sum \bar{x}_{i} \sum \bar{y}_{i}}{n \sum \bar{x}_{i}{ }^{2}-\left(\sum \bar{x}_{i}\right)^{2}}=\frac{\sum \bar{x}_{i} \bar{y}_{i}-n \bar{x} \bar{y}}{\sum \bar{x}_{i}{ }^{2}-n \bar{x}^{2}} \\
b=\frac{\sum \bar{x}_{i}{ }^{2} \sum \bar{y}_{i}-\sum \bar{x}_{i} \sum \bar{x}_{i} \bar{y}_{i}}{n \sum \bar{x}_{i}{ }^{2}-\left(\sum \bar{x}_{i}\right)^{2}} \Leftrightarrow b=\frac{\sum \bar{y}_{i}}{n}-a \frac{\sum \bar{x}_{i}}{n}=\bar{y}-a \bar{x}
\end{array}\right.
$$

onde $\bar{x}$ (respectivamente $\bar{y}$ ) é a média dos valores $\bar{x}_{i}$ (respectivamente $\bar{y}_{i}$ ).

Para modelos dados por outras funções (não lineares), o método do ajuste linear é ainda aplicável, se conseguirmos escrever estas funções na forma:

$$
f(\tau)=\alpha \tau+\beta
$$

com $\alpha$ e $\beta$ números reais, mediante mudança de variável $\tau=g(y)$. No caso é bom considerar as funções típicas: quadrática, exponencial, logarítmico, hiperbólico, geométrico, entre outas.

O ajuste linear do modelo exponencial, no caso da proposta deste trabalho, geralmente emprega uma função do tipo:

$$
y(x)=b e^{a x}, \quad b>0
$$


Se considerarmos a mudança de variável $z=\ln y$, utilizando as propriedades de logaritmos, teremos:

$$
z=a x+\ln b
$$

Se $a>0$, a exponencial será crescente e se $a<0$, decrescente. A equação (7) poderá ser reescrita como:

$$
z=\alpha x+\beta
$$

sendo $z=\ln y, \alpha=a$ e $\beta=\ln b$.

Ao plotarmos o gráfico da proposta em estudo "tempo de exposição segura ao sol utilizando FPS 30, em relação os níveis de radiação ultravioleta $(U V B)$ para cada tipo de pele abordado", observamos que temos uma função que se aproxima do modelo exponencial para o ajuste dos dados, de modo que é preciso linearizá-la antes de aplicar o método dos mínimos quadrados.

A linearização consiste em transformar a equação linear numa equação equivalente à equação exponencial, seu cálculo será facilitado com a mudança de variável $z_{i}=\ln y_{i}$, utilizando a equação (4), temos:

$$
\alpha=\frac{\sum \bar{x}_{i} \bar{z}_{i}-\frac{\sum \bar{x}_{i} \sum \bar{z}_{i}}{n}}{\sum \bar{x}_{i}^{2}-\frac{\left(\sum \bar{x}_{i}\right)^{2}}{n}} \quad e \quad \beta=\frac{\sum \bar{z}_{i}}{n}-\alpha \frac{\sum \bar{x}_{i}}{n}
$$

Observe que a equação (8) é idêntica à equação (2), exceto pelo fato de que a variável é calculada pelo logaritmo de base natural da variável original. Aplicando-se transformações, obtêm-se as constantes de ajuste exponencial ( $\alpha$ e $\beta$ ), e assim empregando a equação (9) do método dos mínimos quadrados para a função linear.

Quando fazemos um ajuste para relacionar duas variáveis, não sabemos se a reta/curva encontrada é de fato o melhor modelo de ajuste. A verificação da existência e do grau de relação entre variáveis é objeto de estudo da correlação. A correlação linear mede a relação existente entre as variáveis $\bar{x}_{i}$ e $\bar{y}_{i}$, dados em torno de uma reta ajustada $y=a x+b$. Para os casos da correlação linear, utiliza-se o Coeficiente da correlação de Pearson $(r)$, dado por: 


$$
r=\frac{\sum_{i=1}^{n} x_{i} y_{i}-\frac{\left(\sum_{i=1}^{n} x_{i}\right)\left(\sum_{i=1}^{n} y_{i}\right)}{n}}{\left\{\left[\sum_{i=1}^{n} x_{i}^{2}-\frac{\left(\sum_{i=1}^{n} x_{i}\right)^{2}}{n}\right]\left[\sum_{i=1}^{n} y_{i}^{2}-\frac{\left(\sum_{i=1}^{n} y_{i}\right)^{2}}{n}\right]\right\}^{1 / 2}}
$$

O intervalo de variação de $r$ é entre $-1 \mathrm{e}+1$, isto é, $-1 \leq r \leq 1$. A correlação será tanto mais forte quanto mais próximo $r$ estiver de \pm 1 , será tanto mais fraca quanto mais próximo estiver de zero. Se $r= \pm 1$, então a correlação entre as variáveis é perfeita. Se $r=0$, então não existe nenhuma correlação. O sinal de $r$ indica o coeficiente angular da reta ajustada.

Para validar o modelo exponencial a ser descrito anteriormente, deve-se fazer ajuste linear do modelo exponencial, sendo $z=\ln y=a x+\ln b$. Com a mudança de variável $z_{i}=\ln y_{i}$, substituída na fórmula do Coeficiente de Correlação de Pearson, temos:

$$
r=\frac{\sum_{i=1}^{n} x_{i} z_{i}-\frac{\left(\sum_{i=1}^{n} x_{i}\right)\left(\sum_{i=1}^{n} z_{i}\right)}{n}}{\left\{\left[\sum_{i=1}^{n} x_{i}^{2}-\frac{\left(\sum_{i=1}^{n} x_{i}\right)^{2}}{n}\right]\left[\sum_{i=1}^{n} z_{i}^{2}-\frac{\left(\sum_{i=1}^{n} z_{i}\right)^{2}}{n}\right]\right\}^{1 / 2}}
$$

O estudo do comportamento das variáveis poderá revelar a existência de uma correlação positiva ou negativa. Se o valor encontrado para a correlação for positivo, significa que uma variável se move no mesmo sentido da outra, não necessariamente, porém, na mesma proporção. Já se o valor da correlação for negativo, significa que uma variável se move em sentido contrário ao da outra, e da mesma forma que a correlação positiva, não necessariamente, porém, na mesma proporção.

O método dos mínimos quadrados pode ser aplicado em várias situações de ensinoaprendizagem, dentre eles aproximar dados obtidos experimentalmente por uma função linear ou não linear, pois apresenta uma forma de ajustar dados de maneira que o erro seja minimizado. Em casos em que o ajuste linear não pode ser considerado, Ruggiero e Lopes (1996) indicam que, para se aplicar o método dos mínimos quadrados, é necessário que se efetue uma linearização do problema através de alguma transformação conveniente, ou seja, que se adapte o caso a um ajuste linear.

Contudo, a formulação de um modelo matemático é geralmente a parte mais difícil de todo o processo de modelagem. Portanto, o professor que estiver desempenhando alguma 
atividade de modelagem matemática pode optar por usar programas computacionais e até mesmo fazer o uso de calculadora científica, que possua este ajuste como opção e também para verificação do cálculo em relação à validade do modelo (correlação). Cumpre destacar, novamente, que o mais importante não é o método para ser chegar ao modelo, mas a realização do processo em si.

\section{TEMPO DE EXPOSIÇÃO AO SOL COM O USO DO FPS 30: UMA PROPOSTA DIDÁTICA PARA O ENSINO DE MATEMÁTICA}

Nessa seção, será apresentada uma sugestão de aula para se trabalhar o método dos mínimos quadrados no ensino médio, em especial no terceiro ano, tendo, como suporte, a utilização do software Excel para a construção dos gráficos. Buscando, assim, contemplar algumas habilidades de matemática e suas tecnologias, conforme preconizado pela Base Nacional Comum Curricular (BNCC), os discentes devem obter a habilidade de "resolver e elaborar problemas do cotidiano, da Matemática e de outras áreas do conhecimento, que envolvem equações lineares simultâneas, usando técnicas algébricas e gráficas, com ou sem apoio de tecnologias digitais" (BRASIL, 2018, p. 536).

O interessante para os encaminhamentos dessa proposta é que o professor deverá somente abordar os principais pontos, ou seja, evidenciar o tópico sobre o tempo de exposição ao sol, suas consequências e apresentar as definições do método de ajuste de curvas. Lembrando que a maior parte do trabalho - a construção do modelo matemático para cada tipo de pele deverá ser desenvolvida pelos alunos, já que o objetivo central da modelagem matemática como metodologia de ensino é colocar os alunos como os sujeitos da construção do conhecimento, com a supervisão e a orientação do professor.

A proposta didática, intitulada "tempo de exposição ao sol com o uso do FPS 30", divide-se em quatro atividades:

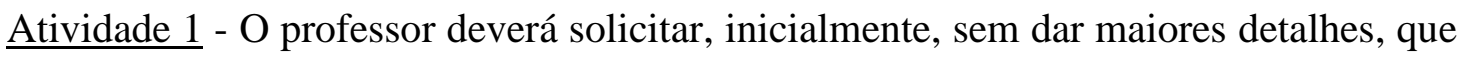
os alunos (grupos) pesquisem (extraclasse) sobre o tempo de exposição segura ao sol, sobre a radiação UV e sobre as formas de proteção e o significado da sigla contida nas embalagens dos protetores solar (FPS). Essa pesquisa pode ser solicitada em forma de perguntas:

- Quanto tempo uma pessoa (não estando em tratamento dermatológico), sem nenhum tipo de proteção, pode permanecer ao sol, sem sofrer queimaduras?

- Qual a variação da radiação solar durante o dia? 
- Qual o significado da sigla contida nas embalagens dos protetores solar (FPS)?

- Quanto tempo uma pessoa (não estando em tratamento dermatológico), utilizando algum FPS, pode permanecer ao sol, sem sofrer queimaduras?

Atividade 2 - Após o tempo despendido para a pesquisa, o professor deverá reservar, pelo menos, uma aula para a discussão em grupo do tema e das variáveis encontradas. No mínimo será encontrada a tabela de exposição segura ao sol em minutos em relação ao tipo de pele (Tabela 2). A partir dessas variáveis, os alunos devem ser solicitados a criar gráficos (extraclasse), com os valores encontrados, que reflitam as variáveis encontradas em relação ao uso do protetor solar. Com a construção desses gráficos, os alunos irão perceber que a curva formada não representa nenhuma das funções já estudadas no primeiro ano do ensino médio (afim, quadrática, exponencial e logarítmica). Por outro lado, os discentes podem se perguntar: qual a curva que melhor representaria esses dados?

$\underline{\text { Atividade } 3}$ - Em outra aula, o professor deve apresentar o método dos mínimos quadrados, sem a utilização da demonstração por derivada, com algumas formas de linearização de funções e fornecer fontes para que os alunos possam encontrar outros tipos de ajustes de curvas, para funções quadráticas, logarítmicas, exponenciais e outras. Assim, após a apresentação do método dos mínimos quadrados, pode-se instigar os grupos, formados de no máximo por três alunos, a criarem um modelo matemático que mostre o tempo que uma pessoa pode ficar exposta ao sol, utilizando algum FPS, tendo por base o método apresentado pelo professor no início desta atividade.

O professor também, além do método dos mínimos quadrados, poderá trabalhar com softwares matemáticos e/ou calculadora científica. Neste trabalho, foi utilizado o Excel para a construção dos gráficos no ajuste de curvas, a partir dos valores informados. Entretanto, para facilitar a aplicabilidade nas escolas da rede pública, é possível adotar outros softwares, de preferência os gratuitos, como por exemplo, o software "LAB Fit ajuste de curvas", desenvolvido pela Universidade Federal de Campina Grande (UFCG). "Trata-se de um pacote de programas envolvendo ajuste de curvas, gráficos 2D e 3D, estatística básica, propagação de erros e algumas ferramentas matemáticas" (SILVA et al., 2004, p. 419).

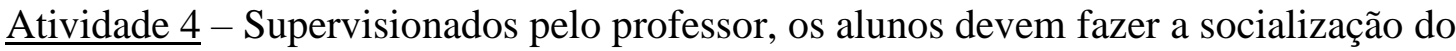
trabalho realizado, com discussão das implicações dos modelos matemáticos elaborados. É importante que os alunos exponham todos os pontos aprendidos. O que se espera: "tempo de exposição segura ao sol; classificação dos tipos de pele; índices de radiação UV (diferenças 
entre UVA, UVB e UVC); o significado da sigla contida nas embalagens dos protetores solar (FPS); tempo de reaplicação do protetor solar; outras medidas de proteção contra a radiação solar, incidência de câncer de pele no país e outros danos causados pela radiação solar, ampliação do conhecimento de funções".

E, após o trabalho, deseja-se que os alunos tenham obtido informações suficientes sobre o tema, a fim de que disseminem esse conhecimento no meio em que estão inseridos, promovendo medidas adequadas para a sua proteção e proteção de terceiros quanto à radiação solar, e que estimulem as mudanças de hábito, o autocuidado, promovendo, assim, a proteção à saúde. Diante de tanta informação que essa abordagem proporciona, cabe ao professor decidir se a atividade será realizada somente nas aulas de matemática, ou se haverá o envolvimento dos demais professores das áreas de biologia, química, física, entre outros.

A seguir apenas uma sugestão para que essa proposta seja realizada, sendo que cumpre ao professor decidir a melhor forma que essa atividade poderá ser trabalhada em sala de aula. Com certeza, além dos modelos matemáticos que serão apresentados, esta pesquisa, pela gama de conhecimentos envolvidos, abre campo para a criação de outros modelos.

A partir da associação do tipo de pele com o tempo que uma pessoa pode ficar exposta ao sol em reação ao índice de radiação (UVB), de acordo com a Tabela 2, será calculado o tempo de exposição que uma pessoa pode permanecer exposta ao sol por tipo de pele, utilizando corretamente FPS 30 em relação ao índice de radiação (UVB). Os resultados de todos os cálculos estão dispostos na Tabela 3.

Tabela 3 - Tempo médio (em horas) de exposição segura ao sol em relação ao índice UVB, utilizando FPS 30

\begin{tabular}{|c|c|c|c|c|c|c|c|c|c|c|c|c|c|c|}
\hline $\begin{array}{c}\text { Valor do } \\
\text { Índice } \\
\text { UVB } \\
\end{array}$ & $0-2$ & 3 & 4 & 5 & 6 & 7 & 8 & 9 & 10 & 11 & 12 & 13 & 14 & 15 \\
\hline $\begin{array}{c}\text { Pele } \\
\text { Tipo A }\end{array}$ & $15 \mathrm{~h}$ & $10 \mathrm{~h}$ & $7,5 \mathrm{~h}$ & $6 h$ & $5 \mathrm{~h}$ & $4,25 \mathrm{~h}$ & $3,75 \mathrm{~h}$ & $3,5 \mathrm{~h}$ & $3 \mathrm{~h}$ & $2,75 \mathrm{~h}$ & $2,5 \mathrm{~h}$ & $2,25 \mathrm{~h}$ & $2 \mathrm{~h}$ & $1,75 \mathrm{~h}$ \\
\hline $\begin{array}{c}\text { Pele } \\
\text { Tipo B }\end{array}$ & $30 \mathrm{~h}$ & $23,5 \mathrm{~h}$ & $16 \mathrm{~h}$ & $12,5 \mathrm{~h}$ & $11 \mathrm{~h}$ & $9,5 \mathrm{~h}$ & $8,5 \mathrm{~h}$ & $7,5 \mathrm{~h}$ & $7 \mathrm{~h}$ & $6,5 \mathrm{~h}$ & $6 h$ & $5,5 \mathrm{~h}$ & $5 \mathrm{~h}$ & $4,5 \mathrm{~h}$ \\
\hline $\begin{array}{c}\text { Pele } \\
\text { Tipo C }\end{array}$ & $45 \mathrm{~h}$ & $35 \mathrm{~h}$ & $25 \mathrm{~h}$ & $20 \mathrm{~h}$ & $17,5 \mathrm{~h}$ & $15 \mathrm{~h}$ & $13 \mathrm{~h}$ & $12 \mathrm{~h}$ & $11 \mathrm{~h}$ & $10 \mathrm{~h}$ & $9,5 \mathrm{~h}$ & $9 \mathrm{~h}$ & $8,5 \mathrm{~h}$ & $7,5 \mathrm{~h}$ \\
\hline $\begin{array}{c}\text { Pele } \\
\text { Tipo D }\end{array}$ & $60 \mathrm{~h}$ & $45 \mathrm{~h}$ & $37,5 \mathrm{~h}$ & $30 \mathrm{~h}$ & $25 \mathrm{~h}$ & $20 \mathrm{~h}$ & $17,5 \mathrm{~h}$ & $16,5 \mathrm{~h}$ & $15 \mathrm{~h}$ & $13,5 \mathrm{~h}$ & $12,5 \mathrm{~h}$ & $11,5 \mathrm{~h}$ & $10,5 \mathrm{~h}$ & $10 \mathrm{~h}$ \\
\hline
\end{tabular}

Fonte: Elaborado pelos autores (Adaptação da Tabela 2).

Tendo por parâmetro os dados da Tabela 3 , foi designado como variável dependente "y" o tempo de exposição segura ao sol, utilizando FPS 30 para cada tipo de pele, e variável independente "x" os índices de radiação UVB. De posse dessas informações e com auxílio de 
um programa computacional (Software Excel), dispomos os dados num sistema de eixos cartesiano, gerando os pontos (diagrama de dispersão) para dar suporte para a construção dos gráficos. Ao construir os gráficos de uma variável em relação à outra, para cada tipo de pele, é possível observar que todos apresentam forma aproximada de uma curva decrescente, e isso se dá pelo fato de que, à medida que o índice de radiação UVB aumenta, o tempo de exposição ao sol diminui. Tem-se aí uma relação de dependência entre duas grandezas (para cada tipo de pele), o que denota o conceito de função.

Como, ao observar os gráficos a seguir, vemos que todos se assemelham ao gráfico de uma função exponencial decrescente, os modelos serão elaborados, considerando o conceito de ajuste linear do modelo exponencial. Contudo, a partir desse momento serão trabalhadas a criação dos modelos e a sua validação (para cada tipo de pele).

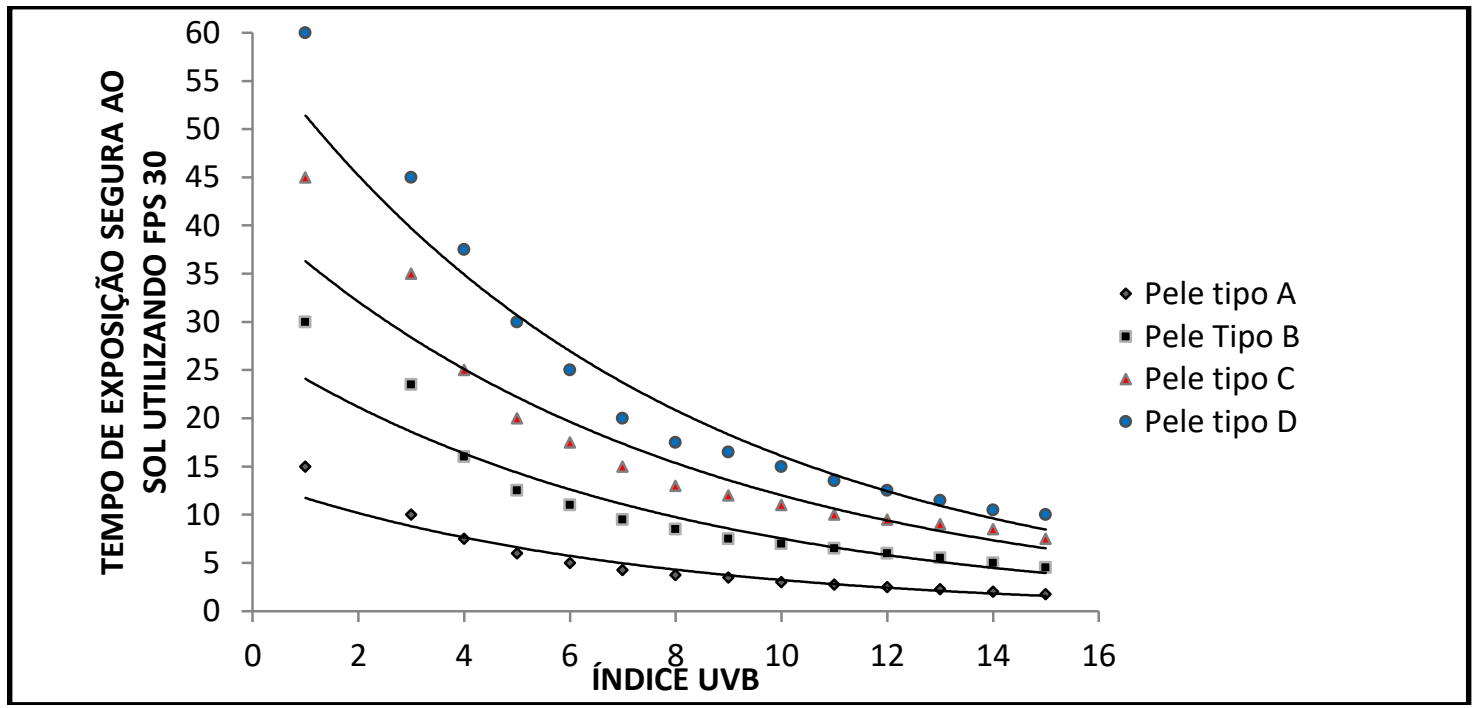

Figura 1 - Gráfico do tempo de exposição ao sol com FPS 30 em relação ao índice UVB para cada tipo de pele. Fonte: Elaborado pelos autores.

\subsection{Desenvolvimento do modelo: tipo de pele A}

Para determinar os modelos matemáticos, será utilizado o método dos mínimos quadrados, de acordo com as equações (8), (9) e (11). Para melhor visualização das informações, serão descritos todos os passos referentes ao tipo de pele A.

De posse das informações obtidas na Tabela 3, vamos representar na Tabela 4 esses dados denotados aqui como sendo variáveis como " $\mathrm{x}$ " e " $\mathrm{y}$ ". 
Tabela 4 - Tempo (em horas) de exposição ao sol com FPS 30 em relação ao índice UVB para o tipo de pele A

\begin{tabular}{c|c|c|c|c|c|c|c|c|c|c|c|c|c|c}
\hline $\mathbf{X}$ & $0-2$ & 3 & 4 & 5 & 6 & 7 & 8 & 9 & 10 & 11 & 12 & 13 & 14 & 15 \\
\hline $\mathbf{Y}$ & 15 & 10 & 7,5 & 6 & 5 & 4,25 & 3,75 & 3,5 & 3 & 2,75 & 2,5 & 2,25 & 2 & 1,75 \\
\hline & \multicolumn{10}{c}{ Fonte: Elaborado peloçãa da Tabela 2). }
\end{tabular}

A partir da Tabela 4 com os dados condensados e usando um programa computacional, segue o gráfico para o tipo de pele A, conforme Figura 2.

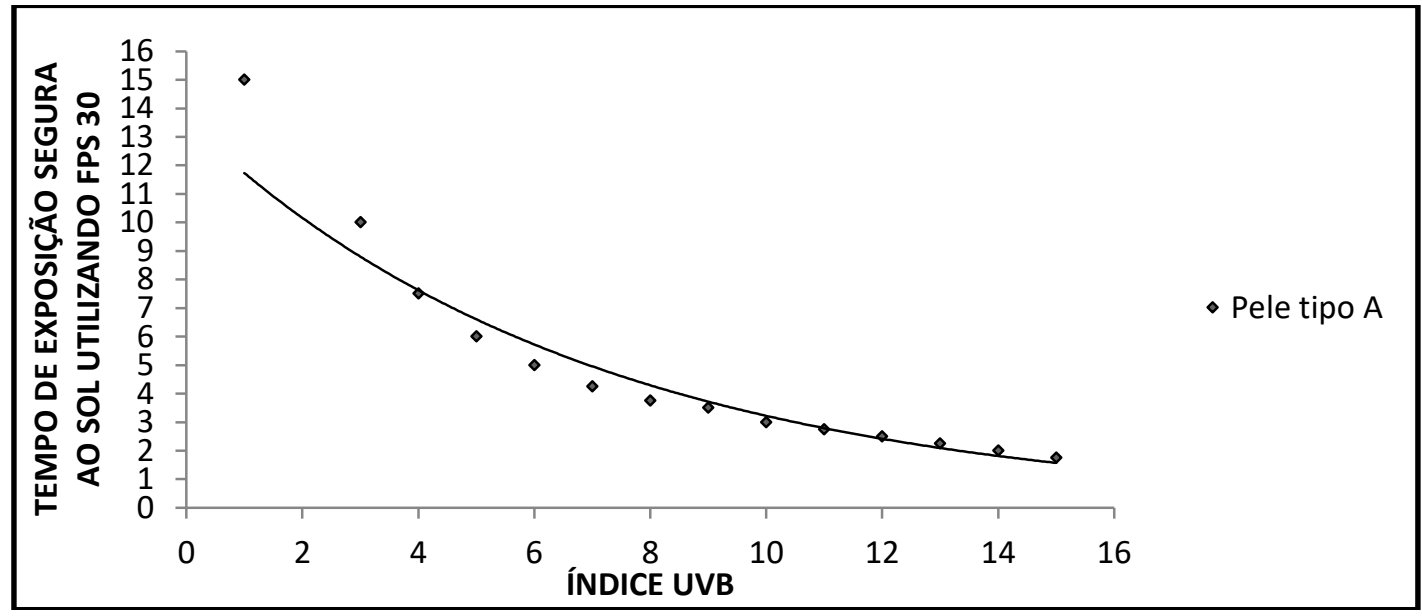

Figura 2 - Tempo (horas) de exposição ao sol utilizando FPS 30 em relação ao índice UVB para o tipo de pele A Fonte: Elaborado pelos autores.

Através da linearização de funções, utilizando o método dos mínimos quadrados, apresentam-se os cálculos para o tipo de pele A. Para introduzir o cálculo do método dos mínimos quadrados nas aulas do ensino médio, indicamos que se trabalhe utilizando somatórias para cada item contido na fórmula, conforme descrito na Tabela 5.

Tabela 5 - Desenvolvimento dos cálculos para elaboração do modelo do tipo de pele A

\begin{tabular}{c|c|c|c|c|c|c}
\hline $\mathbf{N}$ & $\mathbf{x}_{\mathbf{i}}$ & $\mathbf{y}_{\mathbf{i}}$ & $\mathbf{z}_{\mathbf{i}}=\mathbf{l n}\left(\mathbf{y}_{\mathbf{i}}\right)$ & $\mathbf{x}_{\mathbf{i}}^{\mathbf{2}}$ & $\mathbf{X}_{\mathbf{i}} \mathbf{Z}_{\mathbf{i}}$ & $\mathbf{Z}_{\mathbf{i}}^{\mathbf{2}}$ \\
\hline 1 & 1 & 15 & 2,7080502 & 1 & 2,7080502 & 7,3335359 \\
\hline 2 & 3 & 10 & 2,3025851 & 9 & 6,9077553 & 5,3018981 \\
\hline 3 & 4 & 7,5 & 2,0149030 & 16 & 8,0596121 & 4,0598342 \\
\hline 4 & 5 & 6 & 1,7917595 & 25 & 8,9587973 & 3,2104020 \\
\hline 5 & 6 & 5 & 1,6094379 & 36 & 9,6566275 & 2,5902904 \\
\hline 6 & 7 & 4,25 & 1,4469190 & 49 & 10,1284329 & 2,0935745 \\
\hline 7 & 8 & 3,75 & 1,3217558 & 64 & 10,5740467 & 1,7470385 \\
\hline 8 & 9 & 3,5 & 1,2527630 & 81 & 11,2748667 & 1,5694151 \\
\hline 9 & 10 & 3 & 1,0986123 & 100 & 10,9861229 & 1,2069490 \\
\hline 10 & 11 & 2,75 & 1,0116009 & 121 & 11,1276100 & 1,0233364 \\
\hline 11 & 12 & 2,5 & 0,9162907 & 144 & 10,9954888 & 0,8395887 \\
\hline 12 & 13 & 2,25 & 0,8109302 & 169 & 10,5420928 & 0,6576078 \\
\hline 13 & 14 & 2 & 0,6931472 & 196 & 9,7040605 & 0,4804530 \\
\hline 14 & 15 & 1,75 & 0,5596158 & 225 & 8,3942368 & 0,3131698 \\
\hline SOMA & 118 & 69,25 & 19,5383706 & 1236 & 130,0178006 & 32,4270934 \\
\hline \multicolumn{7}{|c|}{ Fonte: Elaborado pelos autores. }
\end{tabular}

Revista REAMEC, Cuiabá (MT), v. 8, n. 2, p. 562-583, maio-agosto, 2020. 
Substituindo os valores encontrados na equação (9), temos:

$$
\begin{gathered}
\alpha=\frac{\sum \bar{x}_{i} \bar{z}_{i}-\frac{\sum \bar{x}_{i} \sum \bar{z}_{i}}{n}}{\sum \bar{x}_{i}^{2}-\frac{\left(\sum \bar{x}_{i}\right)^{2}}{n}}=\frac{(130,0178006)-\frac{(118)(19,5383706)}{14}}{(1236)-\frac{(118)^{2}}{14}} \cong-0,1435735 \\
\beta=\frac{\sum \bar{z}_{i}}{n}-\alpha \frac{\sum \bar{x}_{i}}{n}=\frac{19,5383706}{14}-(-0,1435735) \frac{118}{14}=2,6057176
\end{gathered}
$$

A equação da reta ajustada, equação (8), utilizando os resultados com duas casas decimais, é dada por $z \cong-0,14 x+2,61$.

$\operatorname{Com} \beta=\ln b$ e $a=\alpha$, então a curva exponencial ajustada, utilizando os resultados com duas casas decimais, será:

$$
y=b e^{a x}=13,54 e^{-0,14 x}
$$

observação: Como $b=e^{\beta}, \log _{\mathrm{o}} b=e^{2,6057176} \Rightarrow b \cong 13,54$ e $\alpha=a \Rightarrow a \cong-0,14$.

Portanto, o modelo matemático para o tipo de pele A que mais se aproxima da curva é:

$$
y=13,54 e^{-0,14 x}
$$

Após a elaboração do modelo, torna-se necessária a realização da sua validação para garantir a sua confiabilidade. Logo, será utilizado o Coeficiente de Correlação de Pearson, adaptado para funções exponenciais para validar o modelo descrito em relação ao tempo que uma pessoa de pele tipo A, utilizando corretamente FPS 30, pode permanecer no sol. Usando a equação (11), temos:

$$
r=\frac{(130,0178)-\frac{(118)(19,53837060465)}{14}}{\left\{\left[1236-\frac{118^{2}}{14}\right]\left[32,42709-\frac{19,53837060465^{2}}{14}\right]\right\}^{1 / 2}} \cong-0,98
$$

Portanto, o valor da correlação é $r \cong-0,98$, como o valor está próximo de -1 temos uma correlação forte negativa, ou seja, a medida que o valor do índice UVB aumenta, o tempo (horas) de exposição ao sol diminui. O resultado obtido em $r$ indica que há uma forte correlação entre as variáveis em estudo, logo o modelo encontrado expressa uma boa aproximação com o fenômeno em estudo. 


\subsection{Modelo matemático: tipo de pele B, C e D}

Para o desenvolvimento dos modelos matemáticos dos tipos de pele $\mathrm{B}, \mathrm{C}$ e $\mathrm{D}$, foram utilizados os mesmos critérios adotados para o tipo de pele A. A Tabela 6 apresenta os resultados da equação da reta ajustada, a curva exponencial ajustada e o coeficiente de correlação de Pearson para cada tipo de pele.

Tabela 6 - Modelos matemáticos para os tipos de pele B, C e D

\begin{tabular}{c|c|c|c}
\hline Tipo de Pele & Equação da Reta ajustada & $\begin{array}{c}\text { Curva exponencial } \\
\text { ajustada }\end{array}$ & $\begin{array}{c}\text { Coeficiente de Correlação } \\
\text { de Pearson }\end{array}$ \\
\hline $\mathbf{B}$ & $z \cong-0,13 x+3,31$ & $y=27,41 e^{-0,13 x}$ & $r \cong-0,97$ \\
\hline $\mathbf{C}$ & $z \cong-0,12 x+3,71$ & $y=41,03 e^{-0,12 x}$ & $r \cong-0,97$ \\
\hline $\mathbf{D}$ & $z \cong-0,13 x+4,07$ & $y=58,49 e^{-0,13 x}$ & $r \cong-0,98$ \\
\hline
\end{tabular}

Fonte: Elaborado pelos autores.

A partir da elaboração dos modelos, em que a variável "x" representa os índices de radiação UVB e a variável "y" representa o tempo de exposição segura ao sol, utilizando corretamente FPS 30 para cada tipo de pele, os alunos podem tirar conclusões e debater sobre o tema em estudo e o uso de funções exponenciais.

É interessante que, durante a análise do modelo, seja feita a comparação entre os índices de radiação (UVB), denotado por variável " $x$ " e o tempo de exposição segura ao sol, utilizando FPS 30, variável “y”, obtida pelo modelo. Para fazer essa comparação, basta apenas substituir os valores de "x", dados em estudo, na curva exponencial ajustada para cada tipo de pele, a fim de verificar se, para cada modelo, os valores encontrados de "y" são uma boa aproximação dos dados observados. Isso poderá ser realizado em uma tabela para facilitar a comparação dos dados referentes à Tabela 3.

O que também deve ser discutido, após a formulação dos modelos, é que o tempo de exposição segura ao sol, utilizando FPS 30, varia de 1 hora e 45 minutos até 60 horas. Ressaltando que, durante a exposição ao sol, com altas temperaturas, ocorrem fatores que influenciam na eficácia da proteção, tais como: transpiração/sudorese, nadar, banhar-se, secarse com a toalha, entre outros. Portanto, é sempre necessária a reaplicação do produto para manter sua eficácia. De acordo com as orientações da Sociedade Brasileira de Dermatologia, se houver muita transpiração ou exposição solar prolongada, é recomendada a reaplicação a cada 2 a 3 horas. 


\section{CONSIDERAÇÕES}

A utilização da modelagem matemática no ensino da matemática tende a motivar o aprendizado, desenvolver a criatividade dos alunos e propiciar maior compreensão e interpretação da realidade em que estão inseridos, além de permitir o trabalho coletivo entre educando e educadores, na busca de soluções para o problema proposto. Dessa maneira, ao aplicar uma atividade, como a exposta nesta pesquisa, o educador através da modelagem estará dando a oportunidade ao aluno de vivenciar um ambiente investigativo, incentivando a conscientização dos problemas vivenciados pela sociedade.

No caso do estudo "tempo de exposição ao sol com o uso do FPS 30", o papel da matemática ultrapassa os limites da sala de aula e chega à vida social dos alunos, cumprindo o papel de agente transformador. Ao desenvolver essa atividade proposta e outras relacionadas à modelagem matemática, exige-se o exercício constante, tanto do professor quanto dos alunos, de habilidades na busca, na seleção, na organização, na análise, na interpretação e na validação das informações.

Em conformidade com a modelagem matemática, está o método dos mínimos quadrados que estima a tendência de comportamento das grandezas relacionadas para determinação da função aproximadora. Neste artigo, além de mostrar a importância desse método, evidencia-se a possibilidade de aplicá-lo ainda no ensino médio, e para facilitar o processo de linearização pode-se adotar o software Excel como uma ferramenta de apoio aos estudantes, evitando, assim, expressões matemáticas extensas em sala de aula. Assim sendo, esperamos que a linearização da função exponencial exposta para a resolução da atividade proposta desperte o interesse de educadores e educandos por trabalhar outras atividades que propiciem a busca e as construções dos conceitos para linearização de outros tipos de funções mediante transformações convenientes.

Isso posto, o intuito da apresentação desta proposta é contribuir com a prática dos professores de matemática interessados em aplicar a modelagem matemática como uma ferramenta que pode ser utilizada ao longo das aulas do ensino médio. Essa metodologia, além de dar ênfase aos conteúdos ensinados, por exemplo estudo de funções exponenciais, ela permite que os alunos aprendam de modo significativo, relacionando a matemática com a realidade à sua volta e com outras áreas, e que sejam capazes de aplicar os conhecimentos adquiridos em outras situações-problema. 


\section{REFERÊNCIAS}

BARBOSA, V. C.; BREITSCHAFT, A. M. S. Um aparato experimental para o estudo do princípio de Arquimedes. Revista Brasileira de Ensino de Física, v. 28, n.1, p.115-122, 2006. Disponível em: http://www.scielo.br/pdf/rbef/v28n1/a14v28n1.pdf. Acesso em: 13 jan. 2019.

BASSANEZI, R. C. Ensino-aprendizagem com modelagem matemática. São Paulo: Contexto, 2004.

BASSANEZI, R. C. Ensino-aprendizagem com modelagem matemática: uma nova estratégia. 3. ed., São Paulo: Contexto, 2011.

BIEMBENGUT, M. S.; HEIN, N. Modelagem matemática no ensino. 5. ed. São Paulo: Contexto, 2009.

BRASIL. Lei n. 9.394, de 20 de dezembro de 1996. Estabelece as Diretrizes e Bases da Educação Nacional. Diário Oficial da União, seção 1, ano CXXXIV, n. 248, Brasília, 1996. Disponível em: https://www.planalto.gov.br/ccivil_03/Leis/L9394.htm. Acesso em: 20 fev. 2019.

BRASIL. Ministério de Educação e Cultura. Base Nacional Comum Curricular: Educação é a base, 2018. Disponível em:

http://basenacionalcomum.mec.gov.br/images/BNCC_EI_EF_110518_versaofinal_site.pdf. Acesso em: 15 fev. 2019.

D'AMBROSIO, U. Da realidade à ação: reflexões sobre educação matemática. Campinas: Sammus, 1986.

FITZPATRICK, T. B.; MOSHER, D. B. Pigmentação cutânea e distúrbios do metabolismo da melanina. In: ISSELBACHER, K. J. et al. Medicina interna, 9. ed. Rio de Janeiro:

Guanabara Koogan, p.276-284, 1983.

GOMES, R. Análise e interpretação de dados de pesquisa qualitativa. In: DESLANDES, S. F; GOMES, R.; MINAYO, M. C. S.(org.). Pesquisa social: teoria, método e criatividade. 26. ed. Petrópolis, RJ: Vozes, p. 79-108, 2007.

INPE. Radiação solar, camada de ozônio e saúde humana. Ministério da Ciência, Tecnologia e Informação, 2012. Disponível em: http://satelite.cptec.inpe.br/uv/. Acesso em: 10 fev. 2019.

KIRCHHOFF, V. W. J. H. Ozônio e Radiação UV-B. São José dos Campos, São Paulo: Transtec Editorial, 1995.

MATOS, S. P. Cosmetologia aplicada. 1. ed. São Paulo: Érica, 2014.

MENEZES, R. O.; BRAGA, R. M.; SANTO, A. O. E. Cooperação no desenvolvimento de atividades de modelagem na educação básica. REAMEC - Rede Amazônica de Educação 
em Ciências e Matemática, [S.1.], v. 7, n. 1, p. 147-170, jan./jul. 2019. ISSN 2318-6674. Disponível em: http://periodicoscientificos.ufmt.br/ojs/index.php/reamec/article/view/7966. Acesso em: 08 jan. 2020. Doi: https://doi.org/10.26571/REAMEC.a2019.v7.n1.p147-170.i7966

MEYER, J. F. C. A.; CALDEIRA, A. D.; MALHEIROS, A. P. S. Modelagem em Educação Matemática. 3. ed. Belo Horizonte: Autêntica, 2013.

MILESE, S. S.; GUTERRES, S. S. Fatores determinantes na eficácia de fotoprotetores. Caderno de Farmácia, v. 18, n. 2, p. 81-87, 2002. Disponível em: https://www.lume.ufrgs.br/bitstream/handle/10183/19680/000378373.pdf?sequence=1. Acesso em: 10 jan. 2019.

ROUESSAC, F.; ROUESSAC, A. Chemical analysis, modern instrumentation methods and techniques. New Jersey, USA: John Wiley \& Sons, p. 189, 2000.

RUGGIERO, M. A. G.; LOPES, V. L. da R. Cálculo numérico: aspectos teóricos e computacionais. 2. ed. São Paulo: Pearson Makron Books, 1996.

SCHALKA, S. Influência da quantidade aplicada de protetores solares no fator de proteção solar (FPS): avaliação de dois protetores solares com os mesmos ingredientes em diferentes concentrações. Dissertação (Mestrado) - Faculdade de medicina e dermatologia, Departamento de Dermatologia, Programa de Pós-Graduação em Ciências, São Paulo, 2009. Disponível em: https://teses.usp.br/teses/disponiveis/5/5133/tde-29092009164750/publico/SergioSchalka.pdf. Acesso em: 03 jan. 2019.

SILVA, W. P. et al. "LAB Fit Ajustes de Curvas": um software em português para tratamento de dados experimentais. Revista Brasileira de Ensino de Física, v. 26, n. 4, p. 419-427, 2004. ISSN 1806-9126. Disponível em: https://www.scielo.br/pdf/rbef/v26n4/a18v26n4.pdf. Acesso em: 12 jan. 2020.

Submetido em: 17 de abril de 2020 .

Aprovado em: 07 de junho de 2020. 